PROCEEDINGS OF THE

AMERICAN MATHEMATICAL SOCIETY

Volume 133, Number 1, Pages 155-162

S 0002-9939(04)07495-7

Article electronically published on June 2, 2004

\title{
MAJORIZATION, RANGE INCLUSION, AND FACTORIZATION FOR BOUNDED LINEAR OPERATORS
}

\author{
BRUCE A. BARNES
}

(Communicated by Joseph A. Ball)

\begin{abstract}
In this paper, relationships among the concepts, majorization, range inclusion, and factorization, are studied in a general setting for bounded linear operators. Some applications of these concepts are given.
\end{abstract}

\section{INTRODUCTION}

Throughout, $X, Y, Z$, and $W$ are Banach spaces. We denote the Banach space of all bounded linear operators defined on $X$ with values in $Y$ by $B(X, Y)$, and by $B(X)$ when $X=Y$. For $T \in B(X, Y)$, let $\mathbf{R}(T)$ and $\mathbf{N}(T)$ be the range of $T$ and the null space of $T$, respectively.

Definition 1. Assume that $T \in B(X, Y)$ and $S \in B(X, Z)$. Then $T$ majorizes $S$ if there exists $M>0$ such that

$$
\|S x\| \leq M\|T x\| \text { for all } x \in X \text {. }
$$

Let $T \in B(X, Y)$. Assume that $V \in B(Y, Z)$ and that $S=V T$. Then for all $x \in X,\|S x\| \leq\|V\|\|T x\|$. Thus, $T$ majorizes $S$. The second important relation between two operators studied in this paper is range inclusion, the situation where $\mathbf{R}(S) \subseteq \mathbf{R}(T)$ for $T \in B(X, Y)$ and $S \in B(Z, Y)$. Note that when $U \in B(Z, X)$ with $S=T U$, then $\mathbf{R}(S) \subseteq \mathbf{R}(T)$. When either $S=V T$ or $S=T U$, as above, we say that $S$ factors with respect to $T$.

There are two seminal papers in this area, [D] by Ron Douglas, and Mary Embry's paper [E]. In [D], Douglas proved that when $H$ is a Hilbert space and $S, T \in B(H)$, then the following three conditions are equivalent:

(1) $\mathbf{R}(S) \subseteq \mathbf{R}(T)$;

(2) $T^{*}$ majorizes $S^{*}$;

(3) $S=T U$ for some $U \in B(H)$.

In [E, Embry partially extended Douglas' result to operators $S, T \in B(X)$, where $X$ is a general Banach space (the full extent of Douglas' result need not hold in this situation). The concept " $T$ majorizes $S$ " in Definition 1 is taken from [E].

R. Harte in his book [H] considers these concepts in the general context where $S$ and $T$ are bounded linear operators with possibly different domain and range spaces. Some of Embry's arguments work in this general context. None of these

Received by the editors August 1, 2003 and, in revised form, September 9, 2003.

2000 Mathematics Subject Classification. Primary 47A05.

Key words and phrases. Bounded linear operator, majorization, range inclusion, factorization.

(C)2004 American Mathematical Society 
sources, [D], [E], or $[\mathrm{H}]$, has a complete set of results involving majorization, range inclusion, and factorization. The aim of this paper is to complete the picture of the relationships among these concepts, and to give some applications of these ideas.

\section{MAJORIZATION}

In this section we derive some consequences of the property " $T$ majorizes $S$ ".

Remark 2. Assume $T \in B(X, Y)$. It is straightforward to verify the following two statements:

(1) If $S_{1}, S_{2} \in B(X, Z)$ and $T$ majorizes $S_{1}$ and $S_{2}$, then $T$ majorizes $S_{1}+S_{2}$.

(2) If $S \in B(X, Z), R \in B(Z, W)$ and $T$ majorizes $S$, then $T$ majorizes $R S$.

In particular, when all the operators involved are in $B(X)$, then the set of operators majorized by $T$ is a left ideal of $B(X)$.

Proposition 3. Assume that $T \in B(X, Y)$ and $S \in B(X, Z)$. The following are equivalent:

(1) $T$ majorizes $S$.

(2) There exists $V \in B(\overline{\mathbf{R}(T)}, Z)$ such that $S=V T$.

(3) Whenever $\left\{x_{n}\right\} \subseteq X$ with $\left\|T x_{n}\right\| \rightarrow 0$, then $\left\|S x_{n}\right\| \rightarrow 0$.

Proof. Assume that $T$ majorizes $S$. So $\|S x\| \leq M\|T x\|$ for all $x \in X$. Then clearly, (3) holds. We verify that (2) holds. Define $V: \mathbf{R}(T) \rightarrow Z$ by $V(T x)=S x$. The map $V$ is well defined since $\mathbf{N}(T) \subseteq \mathbf{N}(S)$. Now $\|V(T x)\|=\|S x\| \leq M\|T x\|$. Thus, $V$ has a bounded extension, which we also denote as $V$, on $\overline{\mathbf{R}(T)}$. From the definition of $V, S=V T$.

The facts that $(2) \Longrightarrow(1)$ and $(2) \Longrightarrow(3)$ are both easy to verify.

Assume that the property in (3) holds. Note that this property implies that $\mathbf{N}(T) \subseteq \mathbf{N}(S)$. As above, define $V: \mathbf{R}(T) \rightarrow Z$ by $V(T x)=S x$. As a consequence of the assumption in (3), $V$ is a continuous map. This verifies that (2) holds.

That (1) and (2) in Proposition 3 are equivalent was noted in [E].

Proposition 4. Assume that $T \in B(X, Y)$ with $\mathbf{R}(T)$ closed. If $S \in B(X, Z)$ has $\mathbf{N}(T) \subseteq \mathbf{N}(S)$, then $T$ majorizes $S$.

Proof. Define $\widetilde{S}: X / \mathbf{N}(T) \rightarrow Z$ by $\widetilde{S}(x+\mathbf{N}(T))=S x$, and define $\widetilde{T}: X / \mathbf{N}(T) \rightarrow Y$ by $\widetilde{T}(x+\mathbf{N}(T))=T x$. A direct calculation verifies that $\widetilde{S}$ is bounded. Also, $\widetilde{T}^{-1}: \mathbf{R}(T) \rightarrow X / \mathbf{N}(T)$ is a closed linear operator. So since $\mathbf{R}(T)$ is closed, $\widetilde{T}^{-1}$ is bounded on $\mathbf{R}(T)$ by the Closed Graph Theorem. Suppose $\left\{x_{n}\right\} \subseteq X$ with $\left\|T x_{n}\right\| \rightarrow 0$. Then $\left\|x_{n}+\mathbf{N}(T)\right\|=\left\|\widetilde{T}^{-1}\left(T x_{n}\right)\right\| \rightarrow 0$; so $\left\|S x_{n}\right\|=$ $\left\|\widetilde{S}\left(x_{n}+\mathbf{N}(T)\right)\right\| \rightarrow 0$. Therefore by Proposition 3 (3), $T$ majorizes $S$.

For $T \in B(X)$, let $r(T)=\lim _{n \rightarrow \infty}\left\|T^{n}\right\|^{\frac{1}{n}}$ denote the spectral radius of $T$.

Proposition 5. Assume that $T \in B(X, Y), S \in B(X, Z)$, and that $T$ majorizes $S$, i.e.,

$$
\|S x\| \leq M\|T x\| \text { for all } x \in X .
$$

(1) If $\mathbf{R}(S)$ is closed and $\mathbf{N}(T)=\mathbf{N}(S)$, then $\mathbf{R}(T)$ is closed.

(2) When $T, S \in B(X)$ and $T S=S T$, then $T^{n}$ majorizes $S^{n}$ for $n \geq 1$. Also, $r(S) \leq M r(T)$. So if $T$ is quasinilpotent, then $S$ is quasinilpotent. 
Proof. (1) For $R \in B(X, Y)$, define $Q_{R}: X \rightarrow X / \mathbf{N}(R)$ by $Q_{R}(x)=x+\mathbf{N}(R)$.

As is well known, $R$ has closed range if and only if $\exists m>0$ such that $m\left\|Q_{R}(x)\right\| \leq$ $\|R x\|$ for all $x \in X[\mathrm{G}$, Theorem IV.1.6]. In the terminology of this paper, $\mathbf{R}(R)$ is closed if and only if $R$ majorizes $Q_{R}$. Now assume that $\mathbf{R}(S)$ is closed and that $\mathbf{N}(T)=\mathbf{N}(S)$. Then $Q_{S}=Q_{T}$ and $S$ majorizes $Q_{S}$. Therefore, $T$ majorizes $Q_{T}$; so $\mathbf{R}(T)$ is closed.

(2) First we show by induction that for all $n \geq 1,\left\|S^{n} x\right\| \leq M^{n}\left\|T^{n} x\right\|$ for all $x \in X$. This holds for $n=1$ by hypothesis. If $\left\|S^{m} x\right\| \leq M^{m}\left\|T^{m} x\right\|$ for all $x \in X$, then for all $x$,

$$
\begin{aligned}
\left\|S^{m+1} x\right\| & =\left\|S^{m}(S x)\right\| \leq M^{m}\left\|T^{m}(S x)\right\|=M^{m}\left\|S\left(T^{m} x\right)\right\| \\
& \leq M^{m}\left[M\left\|T\left(T^{m} x\right)\right\|\right]=M^{m+1}\left\|T^{m+1} x\right\| .
\end{aligned}
$$

This completes the induction proof.

It follows from this result that $T^{n}$ majorizes $S^{n}$ for $n \geq 1$. Also, $\left\|S^{n}\right\|^{\frac{1}{n}} \leq$ $M\left\|T^{n}\right\|^{\frac{1}{n}}$ for all $n$; so $r(S) \leq M r(T)$.

Proposition 6. Assume that $T \in B(X, Y), S \in B(X, Z)$, and that $T$ majorizes $S$.

(1) If $T$ is compact, then $S$ is compact.

(2) If $T$ is weakly compact, then $S$ is weakly compact.

(3) If $T$ is strictly singular, then $S$ is strictly singular.

Proof. Since $T$ majorizes $S$, by Proposition 3, there exists $V \in B(\overline{\mathbf{R}(T)}, Z)$ such that $S=V T$. Since the product of a compact operator and a bounded linear operator is compact [DS, Theorem 4, p. 486], (1) follows. The proofs of (2) and (3) are the same, using [DS Theorem 5, p. 484] when $T$ is weakly compact, and using [G, III.2.5, Theorem, p. 87] when $T$ is strictly singular.

\section{DuAl PROperties}

In this section we prove some dual relationships between range inclusion and majorization.

The dual space of $X$ is denoted by $X^{*}$. For $x \in X$ and $\alpha \in X^{*}$, we use the form notation, $\langle x, \alpha\rangle \equiv \alpha(x)$. For $T \in B(X, Y), T^{*} \in B\left(Y^{*}, X^{*}\right)$ is the usual adjoint of $T$. With respect to the form notation, $\langle T x, \alpha\rangle=\left\langle x, T^{*} \alpha\right\rangle$ for all $x \in X$ and all $\alpha \in Y^{*}$.

Theorem 7. (1) Assume that $T \in B(X, Y)$ and $S \in B(X, Z)$, and that $T$ majorizes $S$. Then $\mathbf{R}\left(S^{*}\right) \subseteq \mathbf{R}\left(T^{*}\right)$.

(2) Assume that $T \in B(X, Y)$ and $S \in B(X, Z)$, and that $\mathbf{R}\left(S^{*}\right) \subseteq \mathbf{R}\left(T^{*}\right)$. Then $T$ majorizes $S$.

(3) Assume that $T \in B(X, Y)$ and $S \in B(Z, Y)$, and that $\mathbf{R}(S) \subseteq \mathbf{R}(T)$. Then $T^{*}$ majorizes $S^{*}$.

(4) Assume $X$ is reflexive. Assume that $T \in B(X, Y)$ and $S \in B(Z, Y)$, and that $T^{*}$ majorizes $S^{*}$. Then $\mathbf{R}(S) \subseteq \mathbf{R}(T)$.

Proof. Assume that $T$ majorizes $S$. So by Proposition 3 (2), there exists $V \in$ $B(\overline{\mathbf{R}(T)}, Z)$ such that $S=V T$. Now $S^{*} \in B\left(Z^{*}, X^{*}\right)$ and $T^{*} \in B\left(Y^{*}, X^{*}\right)$. Assume that $\alpha \in Z^{*}$ and consider $S^{*} \alpha$. For all $x \in X,\left\langle x, S^{*} \alpha\right\rangle=\langle S x, \alpha\rangle=$ $\langle V T x, \alpha\rangle=\left\langle T x, V^{*} \alpha\right\rangle$, where $V^{*} \alpha$ is a continuous linear functional on $\overline{\mathbf{R}(T)}$. Let $\beta$ be any extension of $V^{*} \alpha$ to $Y^{*}$ [Hahn-Banach]. Then for all $x \in X,\left\langle x, S^{*} \alpha\right\rangle=$ $\langle T x, \beta\rangle=\left\langle x, T^{*} \beta\right\rangle$. Thus, $S^{*} \alpha=T^{*} \beta$. This shows that $\mathbf{R}\left(S^{*}\right) \subseteq \mathbf{R}\left(T^{*}\right)$. 
Assume the hypotheses in (2). Note that $\mathbf{N}(T) \subseteq \mathbf{N}(S)$. Then the linear map $V: \mathbf{R}(T) \rightarrow Z$ defined by $V(T x)=S x, x \in X$, is well defined. Suppose $V$ is unbounded. Then there exists a sequence $\left\{x_{n}\right\} \subseteq X$ with $\left\|T x_{n}\right\|=1$ for all $n$, and $\left\|S x_{n}\right\| \rightarrow+\infty$. Let $\alpha \in Z^{*}$ be arbitrary, and choose $\beta \in Y^{*}$ such that $S^{*} \alpha=T^{*} \beta$. Then

$$
\left|\left\langle S x_{n}, \alpha\right\rangle\right|=\left|\left\langle x_{n}, S^{*} \alpha\right\rangle\right|=\left|\left\langle x_{n}, T^{*} \beta\right\rangle\right|=\left|\left\langle T x_{n}, \beta\right\rangle\right| \leq\|\beta\| \text { for } n \geq 1 .
$$

It follows from the Uniform Boundedness Principle that $\left\|S x_{n}\right\|$ is bounded, a contradiction. Thus we have that $V$ is bounded on $\mathbf{R}(T)$ and $S=V T$, so $T$ majorizes $S$.

Now assume that $S$ and $T$ are as in (3) with $\mathbf{R}(S) \subseteq \mathbf{R}(T)$. This implies that $\mathbf{N}\left(T^{*}\right) \subseteq \mathbf{N}\left(S^{*}\right)$. Define $U: \mathbf{R}\left(T^{*}\right) \rightarrow Z^{*}$ by $U\left(T^{*} \alpha\right)=S^{*} \alpha$ for all $\alpha \in Y^{*}$ (note that this map is well defined). If $U$ is unbounded, then there exists $\left\{\alpha_{n}\right\} \subseteq Y^{*}$ such that $\left\|T^{*} \alpha_{n}\right\|=1$ for all $n$, while $\left\|S^{*} \alpha_{n}\right\|=\left\|U\left(T^{*} \alpha_{n}\right)\right\| \rightarrow+\infty$. For an arbitrary $z \in Z$, choose $x \in X$ such that $S z=T x$. Then $\left|\left\langle z, S^{*} \alpha_{n}\right\rangle\right|=\left|\left\langle S z, \alpha_{n}\right\rangle\right|=$ $\left|\left\langle T x, \alpha_{n}\right\rangle\right|=\left|\left\langle x, T^{*} \alpha_{n}\right\rangle\right| \leq\|x\|$. Again, from the Uniform Boundedness Principle, $\left\|S^{*} \alpha_{n}\right\|$ is a bounded sequence. This contradiction proves that $U$ is bounded. Therefore, since $S^{*}=U T^{*}, T^{*}$ majorizes $S^{*}$.

Assume the hypotheses in (4). By Proposition 3, $S^{*}=V T^{*}$ where $V: \overline{\mathbf{R}\left(T^{*}\right)} \rightarrow$ $Z^{*}$ is a bounded linear map. Then $V^{*}: Z^{* *} \rightarrow{\overline{\mathbf{R}\left(T^{*}\right)}}^{*} \subseteq X^{* *}$. Assume that $z \in Z$ is arbitrary. Then $z \in Z^{* *}$ and $V^{*} z \in{\overline{\mathbf{R}\left(T^{*}\right)}}^{*}$. Let $\beta$ be any extension of $V^{*} z$ to $X^{* *}$. Since $X$ is reflexive, $\exists y \in X$ such that $\langle y, \delta\rangle=\langle\beta, \delta\rangle$ for all $\delta \in X^{*}$. For all $\alpha \in Y^{*}$,

$$
\langle S z, \alpha\rangle=\left\langle z, S^{*} \alpha\right\rangle=\left\langle z, V T^{*} \alpha\right\rangle=\left\langle V^{*} z, T^{*} \alpha\right\rangle=\left\langle y, T^{*} \alpha\right\rangle=\langle T y, \alpha\rangle .
$$

Therefore $S z=T y$; so $\mathbf{R}(S) \subseteq \mathbf{R}(T)$.

That (1) and (2) in Theorem 7 are equivalent was proved in [E] for $T, S \in B(X)$. Part (3) is proved in $[\mathrm{H}]$ Theorem 10.5.5]. The proof given here is new.

The dual properties in the theorem above combined with Proposition 6 yield the following results concerning range inclusion.

Proposition 8. Assume that $T \in B(X, Y)$ and $S \in B(Z, Y)$.

(1) If $\mathbf{R}(S) \subseteq \mathbf{R}(T)$ and $T$ is compact, then $S$ is compact.

(2) If $\mathbf{R}(S) \subseteq \mathbf{R}(T)$ and $T$ is weakly compact, then $S$ is weakly compact.

(3) If $\mathbf{R}(S) \subseteq \mathbf{R}(T)$ and $T^{*}$ is strictly singular, then $S^{*}$ is strictly singular.

(4) Also, assume that $T \in B(X, Y)$ and $S \in B(X, Z)$. If $\mathbf{R}\left(S^{*}\right) \subseteq \mathbf{R}\left(T^{*}\right)$ and $T$ is strictly singular, then $S$ is strictly singular.

Proof. Assume the hypotheses in (1). Since $\mathbf{R}(S) \subseteq \mathbf{R}(T)$, by Theorem 7 (3), $T^{*}$ majorizes $S^{*}$. Now $T^{*}$ is compact, and so from Proposition $6(1), S^{*}$ is compact. Therefore $S$ is compact by Schauder's Theorem [DS, Theorem 2, p. 485].

The proof of (2) is essentially the same as the proof of (1), using the facts that $T$ is weakly compact if and only if $T^{*}$ is weakly compact [DS, Theorem 8, p. 485]. The proof of (3) also follows the same pattern.

Now assume the hypotheses in (4). Since $\mathbf{R}\left(S^{*}\right) \subseteq \mathbf{R}\left(T^{*}\right)$, by Theorem 7 (2), $T$ majorizes $S$. Then the result follows by applying Proposition 6 (3).

Part (1) of Proposition 8 is known; see BMSW, Corollary O.4.2] for example. The proof given here is new. 


\section{Quasinilpotents; Riesz operators}

In this section we prove some majorization and range inclusion results for two important classes of bounded linear operators, quasinilpotent operators and Riesz operators.

Theorem 9. Assume that $T, S \in B(X), T S=S T$, and that $T$ is quasinilpotent.

(1) If $\mathbf{R}(S) \subseteq \mathbf{R}(T)$, then $S$ is quasinilpotent.

(2) If $T$ majorizes $S$, then $S$ is quasinilpotent.

Proof. We have already proved that (2) holds [Proposition 5, part (2)].

Now suppose that $\mathbf{R}(S) \subseteq \mathbf{R}(T)$. Then by Theorem 7(3), $T^{*}$ majorizes $S^{*}$. Also, $T^{*} S^{*}=S^{*} T^{*}$ and $T^{*}$ is quasinilpotent. By part (2), $S^{*}$ is quasinilpotent; so $S$ is quasinilpotent.

Part (1) of Theorem 9 is known, and is due to M. J. Ganly; see [BMSW O.4.4]. The proof presented here is new.

Let $\ell^{\infty}(X)$ denote the space of all bounded sequences $\left\{x_{n}\right\}_{n \geq 1} \subseteq X$ equipped with the sup-norm, $\left\|\left\{x_{n}\right\}\right\|_{\infty}=\sup \left\{\left\|x_{n}\right\|: n \geq 1\right\}$. For $T \in B(X)$, define $T_{\infty}$ on $\ell^{\infty}(X)$ by $T_{\infty}\left(\left\{x_{n}\right\}\right)=\left\{T x_{n}\right\}$. Let $M$ be the closed subspace of $\ell^{\infty}(X)$ consisting of all sequences $\left\{x_{n}\right\} \in \ell^{\infty}(X)$ with the property that every subsequence of $\left\{x_{n}\right\}$ contains a convergent subsequence. Note that for all $T \in B(X), T_{\infty}(M) \subseteq M$. Let $\widehat{X}$ be the quotient space $\ell^{\infty}(X) / M$, and define $\widehat{T} \in B(\widehat{X})$ by $\widehat{T}\left(\left\{x_{n}\right\}+M\right)=$ $\left\{T x_{n}\right\}+M$. The kernal of the map $T \rightarrow \widehat{T}$ is $K(X)$, the space of all compact linear operators on $X$. Information concerning the representation $T \rightarrow \widehat{T}$ can be found in [BMSW, Section O.2].

Proposition 10. Assume that $T, S \in B(X)$ with $\mathbf{R}(S) \subseteq \mathbf{R}(T)$. Then

$$
\mathbf{R}(\widehat{S}) \subseteq \mathbf{R}(\widehat{T}) .
$$

Proof. We prove that $\mathbf{R}\left(S_{\infty}\right) \subseteq \mathbf{R}\left(T_{\infty}\right)$. From this it follows easily that $\mathbf{R}(\widehat{S}) \subseteq$ $\mathbf{R}(\widehat{T})$. Define $\widetilde{T}: X / \mathbf{N}(T) \rightarrow X$ by $\widetilde{T}(x+\mathbf{N}(T))=T x$. Then $\widetilde{T}^{-1}$ with domain $\mathbf{R}(T) \subseteq X$ is a closed linear operator. Since $\mathbf{R}(S) \subseteq \mathbf{R}(T)$, it follows that $\widetilde{T}^{-1} S$ : $X \rightarrow X / \mathbf{N}(T)$ is closed, and therefore bounded by the Closed Graph Theorem.

Verification that $\widetilde{T}^{-1} S$ is closed: Assume that $z_{n} \rightarrow z_{0}$ in $X$ and $\widetilde{T}^{-1} S\left(z_{n}\right) \rightarrow$ $x_{0}+\mathbf{N}(T)$ in $X / \mathbf{N}(T)$. Then $S\left(z_{n}\right) \rightarrow S\left(z_{0}\right)$ in $\mathbf{R}(T)$, and since $\widetilde{T}^{-1}$ is closed, $\widetilde{T}^{-1} S\left(z_{0}\right)=x_{0}+\mathbf{N}(T)$. Thus, $\widetilde{T}^{-1} S$ is closed.

Now assume that $\left\{z_{n}\right\} \subseteq X$ with $\left\|z_{n}\right\| \leq J$ for all $n$. Let $y_{n}+\mathbf{N}(T)=\widetilde{T}^{-1} S\left(z_{n}\right)$ for all $n$. Setting $M=\left\|\widetilde{T}^{-1} S\right\|$, we have $\left\|y_{n}+\mathbf{N}(T)\right\| \leq M J$ for all $n$. For each $n$, choose $w_{n} \in \mathbf{N}(T)$ such that $\left\|y_{n}+w_{n}\right\| \leq M J+1$. Then $T\left(y_{n}+w_{n}\right)=$ $\widetilde{T}\left(y_{n}+w_{n}+\mathbf{N}(T)\right)=S\left(z_{n}\right)$ for all $n$. This proves that $\mathbf{R}\left(S_{\infty}\right) \subseteq \mathbf{R}\left(T_{\infty}\right)$.

Let $\Phi(X)$ be the set of all Fredholm operators on $X$. We denote the Fredholm spectrum of an operator $T \in B(X)$ by $\sigma_{F}(T)\left[\sigma_{F}(T)=\{\lambda \in \mathbf{C}:(\lambda-T) \notin \Phi(X)\}\right]$.

A key result that we use in what follows is a part of [BMSW, Theorem 0.2.2]:

$$
T \in \Phi(X) \Leftrightarrow \widehat{T} \text { is invertible in } B(\widehat{X}) .
$$

It follows from this that for $T \in B(X), \sigma_{F}(T)=\sigma(\widehat{T})[\sigma(\widehat{T})$ is the usual spectrum of the operator $\widehat{T}$ relative to $B(\widehat{X})]$. 
A linear operator $T \in B(X)$ is a Riesz operator if for all $\lambda \in \mathbf{C}, \lambda \neq 0,(\lambda-T) \in$ $\Phi(X)$. Equivalently, $T \in B(X)$ is a Riesz operator exactly when $\sigma_{F}(T)=\{0\}$. Thus from the discussion above, $T$ is a Riesz operator if and only if $\sigma(\widehat{T})=\{0\}$, i.e., $\widehat{T}$ is quasinilpotent on $\widehat{X}$. It is true that $T \in B(X)$ is a Riesz operator if and only if $T^{*}$ is a Riesz operator. Riesz operators play an important role in the theory of linear operators; information concerning Riesz operators can be found in many books on general operator theory, for example in Chapter 3 of [CPY].

Theorem 11. Assume that $T, S \in B(X), T S-S T$ is compact, and that $T$ is a Riesz operator.

(1) If $\mathbf{R}(S) \subseteq \mathbf{R}(T)$, then $S$ is a Riesz operator.

(2) If $T$ majorizes $S$, then $S$ is a Riesz operator.

Proof. To prove (1), by Proposition 10, $\mathbf{R}(S) \subseteq \mathbf{R}(T)$ implies $\mathbf{R}(\widehat{S}) \subseteq \mathbf{R}(\widehat{T})$. Also, we have $\widehat{S} \widehat{T}=\widehat{T} \widehat{S}$ and $\widehat{T}$ is quasinilpotent. Therefore by Theorem $9, \widehat{S}$ is quasinilpotent; so $S$ is a Riesz operator.

Now assume as in (2) that $T$ majorizes $S$. By Theorem $7(1), \mathbf{R}\left(S^{*}\right) \subseteq \mathbf{R}\left(T^{*}\right)$. Also, $T^{*} S^{*}-S^{*} T^{*}$ is a compact operator. Finally, note that $T^{*}$ is a Riesz operator. Applying part (1), we have that $S^{*}$ is a Riesz operator. It follows that $S$ is a Riesz operator.

Part (1) of Theorem 11 is known; see [BMSW] Theorem O.4.5, p. 14]. The proof given here is new.

\section{FACTORIZATION}

Assume that $T \in B(X, Y)$ and $S \in B(X, Z)$. Then $S$ is a left multiple of $T$ if there exists $V \in B(Y, Z)$ with $S=V T$. There is a similar terminology for when $S$ is a right multiple of $T, S=T U$. In either case we say that $S$ factors with respect to $T$.

Remark 12. A linear operator $T \in B(X, Y)$ has a g-inverse (generalized inverse) $R \in B(Y, X)$ if $T=T R T$. As is well known, in this case $T R \in B(Y)$ is a projection onto $\mathbf{R}(T)$ and $I-R T \in B(X)$ is a projection onto $\mathbf{N}(T)$; see [LT, Theorem 12.9, p. 251]. If $S \in B(X, Z)$ has $\mathbf{N}(T) \subseteq \mathbf{N}(S)$, then $S(I-R T)=0$; so $S=(S R) T$. If $S \in B(Z, Y)$ and $\mathbf{R}(S) \subseteq \mathbf{R}(T)$, then $T(R S)=S$. Thus, $S$ is a left multiple of $T$ in the first case, and a right multiple of $T$ in the second.

In the next result, we look at more general situations where $S$ factors with respect to $T$. Recall that a closed subspace $M$ of $X$ is complemented if there exists a closed subspace $N$ of $X$ such that $X=M \oplus N$.

Theorem 13. Assume $T \in B(X, Y)$.

(1) If $S \in B(X, Z)$ is majorized by $T$ and $\overline{\mathbf{R}(T)}$ is complemented, then there exists $V \in B(Y, Z)$ such that $S=V T$.

(2) If $S \in B(Z, Y)$ with $\mathbf{R}(S) \subseteq \mathbf{R}(T)$ and $\mathbf{N}(T)$ is complemented, then there exists $U \in B(Z, X)$ such that $S=T U$.

(3) If $S \in B(X, Z)$ with $\mathbf{R}\left(S^{*}\right) \subseteq \mathbf{R}\left(T^{*}\right)$ and $\overline{\mathbf{R}(T)}$ is complemented, then there exists $V \in B(Y, Z)$ such that $S=V T$.

(4) Assume $X$ is reflexive. If $S \in B(Z, Y), T^{*}$ majorizes $S^{*}$, and $\mathbf{N}(T)$ is complemented, then there exists $U \in B(Z, X)$ such that $S=T U$. 
Proof. (1) follows from part (2) of Proposition 3 (simply extend $V$ to be the zero operator on a closed complement of $\overline{\mathbf{R}(T)}$ ).

Now assume the hypotheses in (2). Let $W$ be a closed subspace of $X$ with $X=W \oplus \mathbf{N}(T)$. Let $\widetilde{T}: W \rightarrow Y$ be the restriction of $T$ to $W$. Now $\widetilde{T}^{-1}$ is a closed linear map on $\mathbf{R}(T)$, and since $\mathbf{R}(S) \subseteq \mathbf{R}(T), \widetilde{T}^{-1} S: Z \rightarrow W$ is a closed, hence bounded operator by the Closed Graph Theorem. We may consider $U \equiv \widetilde{T}^{-1} S$ as an operator in $B(Z, X)$. Clearly, $S=T \widetilde{T}^{-1} S=T U$.

(3) follows from Theorem 7 (2) and part (1).

(4) follows from Theorem 7 (4) and part (2).

There are some factorization results proved in [H, Theorems 10.2 and 10.4]. A limitation of the results in $[\mathrm{H}]$ is that when the underlying spaces are complete, the given hypotheses imply that $\mathbf{R}(T)$ is closed.

Now we give a sample application in the context of $B(X)$. Let $X_{m}=X \oplus X \oplus$ $\cdots \oplus X$ ( $m$ copies) with norm $\left\|x_{1} \oplus x_{2} \oplus \cdots \oplus x_{m}\right\|=\sum_{k=1}^{m}\left\|x_{k}\right\|$. Note that if $V \in B\left(X_{m}, X\right)$, then $V$ has the form $V\left(x_{1} \oplus x_{2} \oplus \cdots \oplus x_{m}\right)=\sum_{k=1}^{m} V_{k}\left(x_{k}\right)$ where $V_{k} \in B(X)$ for all $k$.

Proposition 14. Assume that $S \in B(X)$, that $T_{k} \in B(X)$ for $1 \leq k \leq m$, and that $\overline{\mathbf{R}\left(T_{k}\right)}$ is complemented for all $k$. Assume that one of the conditions (i) or (ii) given below holds.

(i) $\exists M>0$ such that $\|S x\| \leq M \sum_{k=1}^{m}\left\|T_{k} x\right\|$ for all $x \in X$;

(ii) $\mathbf{R}\left(S^{*}\right) \subseteq \sum_{k=1}^{m} \mathbf{R}\left(T_{k}^{*}\right)$.

Then there exist $V_{k} \in B(X), 1 \leq k \leq m$, such that

$$
S=V_{1} T_{1}+V_{2} T_{2}+\cdots+V_{m} T_{m} .
$$

Proof. Define $T \in B\left(X, X_{m}\right)$ by $T(x)=T_{1} x \oplus T_{2} x \oplus \cdots \oplus T_{m} x, x \in X$. Note that $\overline{\mathbf{R}(T)}$ is complemented since $\overline{\mathbf{R}\left(T_{k}\right)}$ is complemented for all $k$. Applying Theorem 13 (1) if (i) holds or Theorem 13 (3) if (ii) holds, there exists $V \in B\left(X_{m}, X\right)$ such that $S=V T$. As noted above, there exist $V_{k} \in B(X), 1 \leq k \leq m$, such that for all $x \in X$,

$$
S x=V T x=V\left(T_{1} x \oplus T_{2} x \oplus \cdots \oplus T_{m} x\right)=V_{1} T_{1}(x)+V_{2} T_{2}(x)+\cdots+V_{m} T_{m}(x) .
$$

Let $T_{k} \in B(X)$ for $1 \leq k \leq m$. Then $\left\{T_{1}, T_{2}, \ldots, T_{m}\right\}$ is jointly bounded below if $\exists M>0$ such that $\|x\| \leq M \sum_{k=1}^{m}\left\|T_{k} x\right\|$ for all $x \in X$. The following corollary is a direct application of Proposition 14 (taking $S=I$ ).

Corollary 15. Assume that $T_{k} \in B(X)$ for $1 \leq k \leq m$, and that $\overline{\mathbf{R}\left(T_{k}\right)}$ is complemented for all $k$. If $\left\{T_{1}, T_{2}, \ldots, T_{m}\right\}$ is jointly bounded below, then there exist $V_{k} \in B(X), 1 \leq k \leq m$, such that

$$
I=V_{1} T_{1}+V_{2} T_{2}+\cdots+V_{m} T_{m} .
$$

\section{OTHER DIRECTIONS}

In addition to the generalization of R. Douglas' original result in $\mathrm{D}$. to a form that applies to linear operators between Banach spaces, there have been many other generalizations, applications, and variations given of this result. We briefly mention a number of these. 
There is an interesting discussion concerning the relationship between majorization and factorization in general $\mathrm{C}^{*}$-algebras in L. Fialkow's article $[\mathrm{F}]$.

There has been work on factorization in the context of nest algebras; see, for example, $\mathrm{BG}$ ] and $\mathrm{KMT}$.

There has been work involving more structured generalizations of the Douglas result; see, for example, $\mathrm{B}$ ] and Leech's Theorem and related results in [RR.

\section{REFERENCES}

[B] J. Ball, Interpolation problems and Toeplitz operators on multiply connected domains, Integral Eqs. and Operator Theory, 4 (1981), 172-184. MR82i:47036

[BG] J. Ball and I. Gohberg, A commutant lifting theorem for triangular matrices with diverse applications, Integral Eqs. and Operator Theory, 8 (1985), 205-267. MR86i:47006

[BMSW] B. Barnes, G. Murphy, R. Smyth, and T. T. West, Riesz and Fredholm Theory in Banach Algebras, Research Notes in Math. 67, Pitman, Boston, 1982. MR84a:46108

[CPY] S. Caradus, W. Pfaffenberger, and B. Yood, Calkin Algebras and Algebras of Operators on Banach Spaces, Lecture Notes in Pure and Applied Math., Vol. 9, Marcel Dekker, New York, 1974. MR 54:3434

[D] R. Douglas, On majorization, factorization, and range inclusion of operators on Hilbert space, Proc. Amer. Math. Soc. 17 (1966), 413-415. MF 34:3315

[DS] N. Dunford and J. Schwartz, Linear Operators, Part I, Interscience, New York, 1964. MR 90g:47001a

[E] M. Embry, Factorization of operators on a Banach space, Proc. Amer. Math. Soc. 38 (1973), 587-590. MR 47:849

[F] L. Fialkow, Structural properties of elementary operators, Elementary Operators \& Applications, M. Mathieu, Editor, World Scientific, Singapore, 1992. MR93i:47042

[G] S. Goldberg, Unbounded Linear Operators, McGraw-Hill, New York, 1966. MR34:580

$[\mathrm{H}] \quad$ R. Harte, Invertibility and Singularity for Bounded Linear Operators, Pure and Applied Math., Marcel Dekker, New York and Basel, 1988. MR 89d:47001

[KMT] E. Katsoulis, R. Moore, and T. Trent, Interpolation in nest algebras and applications to operator corona theorems, J. Operator Theory 29 (1983), 115-123. MR95b:47052

[LT] D. Lay and A. Taylor, Introduction to Functional Analysis, John Wiley and Sons, New York, 1980. MR81b:46001

[RR] M. Rosenblum and J. Rovnyak, Hardy Classes and Operator Theory, Dover, Mineola, New York, 1997. MR97j:47002

Department of Mathematics, University of Oregon, Eugene, Oregon 97403

E-mail address: barnes@darkwing.uoregon.edu 